\title{
Electronic Biasing of Monolayer Transition Metal Dichalcogenides in a TEM
}

Akshay A. Murthy ${ }^{1,2}$, Teodor K. Stanev ${ }^{3}$, Roberto dos Reis ${ }^{1,4}$, Nathaniel P. Stern ${ }^{3}$ and Vinayak P. Dravid $^{1,2,4 *}$

${ }^{1 .}$ Department of Materials Science and Engineering, Northwestern University, Evanston, USA.

2. International Institute of Nanotechnology, Northwestern University, Evanston, USA.

3. Department of Physics and Astronomy, Northwestern University, Evanston, USA.

4. NUANCE Center, Northwestern University, Evanston, USA.

* Corresponding author: v-dravid@northwestern.edu

Monolayer transition metal dichalcogenides (TMDs), such as $\mathrm{MoS}_{2}$, display intriguing optical and electronic properties including a direct band gap, reasonably high carrier mobilities, and large on/off ratios $[1,2]$. Moreover, by interfacing these materials with one another and introducing abrupt changes in the electronic structure, new characteristics can be unlocked. Several research groups have leveraged this approach to build various optoelectronic nanoarchitectures spanning from atomically thin diodes [3] to photodetectors [4] to photovoltaic cells [5]. Despite the performance of these devices relying solely on the charge transport across the interfacial regions, there remain many unanswered questions with respect to the atomistic dynamics across this localized area. To address this limitation and directly ascertain how interfacial atoms respond to external stimuli, we have constructed a setup for applying a lateral electric field across a 2D material sample that allows for concurrent TEM imaging and associated techniques. Using this platform, we are able to monitor the potential evolution of grain boundary structure in the presence of applied electric fields.

While in situ electrochemical experiments that aim to understand structural dynamics at electrode interfaces have gained great popularity, in situ biasing has been largely unexplored within the 2D materials community. This is primarily a result of the challenges linked to utilizing traditional TEM biasing techniques, such as direct probing of the active material, with atomically thin structures. To construct our setup, we first use a standard polycarbonate-based transfer technique [6] to pick up thermally evaporated gold interdigitated electrodes. These electrodes are transferred onto a $\mathrm{SiO}_{2}$ TEM window grid (TEMWindows.com) with the interdigitated regions sitting across the window region. We then repeat this process with chemical vapor deposited monolayer TMDs and transfer them such that they are supported by the interdigitated electrodes (Figure 1). External wires are bonded from the contact pads to a Nanofactory holder. As such, it is possible to use an external power source to apply a lateral electric field across suspended regions of TMD and image transient processes across various 2D interfaces, including grain boundaries between adjacent monolayer crystals.

Through a combination of real-space and reciprocal space information, the impact of the electric field on grain boundary dynamics can be examined. Figure 2 a demonstrates the relationship between applied voltage and the current response across a monolayer $\mathrm{MoS}_{2}$ grain boundary when measured inside the TEM. In Figures $2 b$ and 2c, TEM images and the associated fast Fourier transform patterns of a low angle grain boundary (indicated by arrows) between adjacent $\mathrm{MoS}_{2}$ crystals are shown. Based on the structural similarity between the pristine (Figure 2b) and biased states (Figure 2c) in both real and reciprocal space representations, it is apparent that grain boundaries within these 2D systems retain structural integrity when subjected to fields on the order of $10^{3} \mathrm{~V} / \mathrm{cm}$. The ongoing measurements include monitoring the details of grain boundary structure, chemistry and some aspects of its electronic structure under applied 
bias, across multiple types of interfaces. We believe this platform offers the potential for new insight that can be utilized to improve existing 2D electronics [7].

\section{References:}

[1] A Splendiani et al, Nano Letters, 10 (2010). p. 1271-1275.

[2] B Radisavljevic et al, Nature Nanotechnology, 6 (2011). p. 147.

[3] MY Li et al, Science 349 (2015). p. 524-528.

[4] Y Li et al, ACS Nano, 11 (2017). p. 10321-10329.

[5] CH Lee et al, Nature Nanotechnology 9 (2014). p. 676.

[6] A Murthy et al, Nano Letters, 18 (2018), p. 2990-2998.

[7] This material is based upon work supported by the NSF (DMR-1507810) and made use of the EPIC facility of Northwestern University's NUANCE Center, which has received support from the Soft and Hybrid Nanotechnology Experimental (SHyNE) Resource (NSF ECCS-1542205); the MRSEC program (NSF DMR-1720319) at the Materials Research Center; the International Institute for Nanotechnology (IIN); the Keck Foundation; and the State of Illinois, through the IIN. A.A.M. gratefully acknowledges support from the Ryan Fellowship and the IIN at Northwestern University.
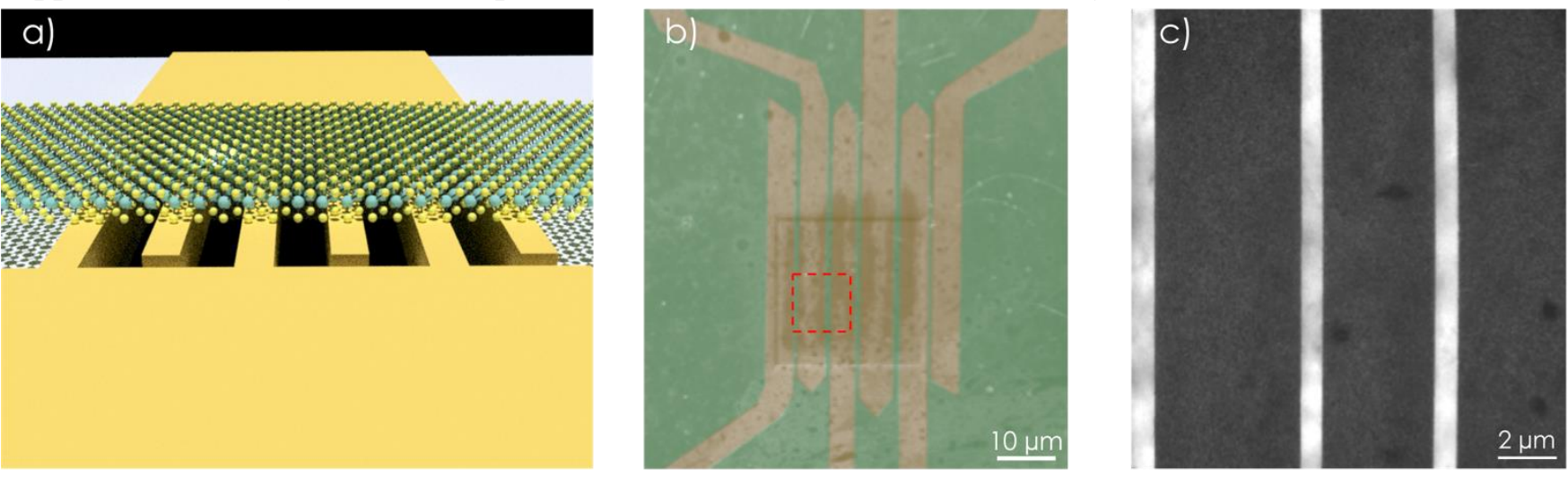

Figure 1. a) Schematic of TEM biasing setup. 2D material is suspended using metal electrodes. b) Low magnification, colored SEM image of sample. Gold color represents metal electrodes and green represents $\mathrm{MoS}_{2}$. Porous region in substrate is beveled for emphasis. c) Low magnification TEM image of sample taken from red boxed region in (b). Light regions represent suspended material, while dark regions represent metal electrodes.
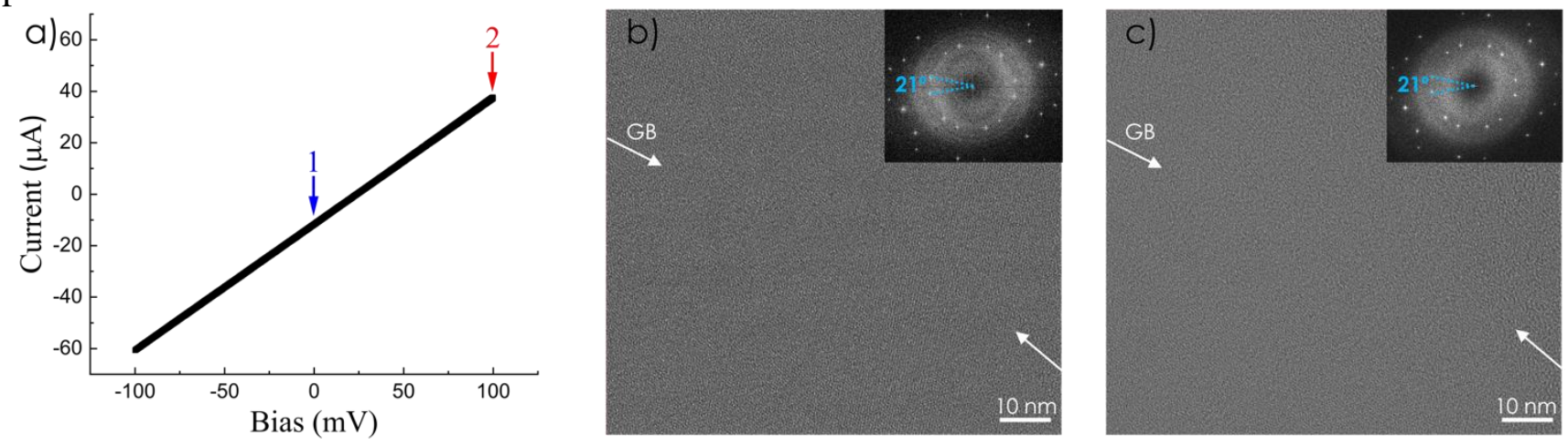

Figure 2. a) Current-Voltage characteristics for the examined material, which compare nicely with prior studies [6]. Imaging was conducted in zero bias state (indicated by blue arrow) and applied bias state (indicated by red arrow). Similarity between HRTEM images of low angle grain boundary (misorientation angle $=21$ degrees - see insets) under (b) zero bias and (c) applied bias state are indicative of overall structural retention. 